\title{
Cromoblastomicosis: características clínicas y microbiológicas de una enfermedad desatendida
}

\author{
Roberto Ventura-Flores, Virgilio Failoc-Rojas y Heber Silva-Díaz
}

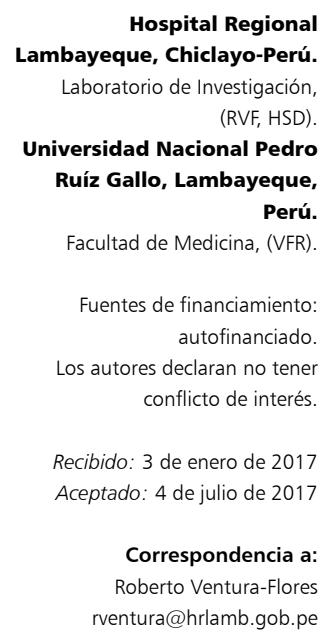

\section{Introducción}

L a cromoblastomicosis, también conocida como cromomicosis, es una infección fúngica, rara, subcutánea crónica, ocasionada por traumatismos o inoculación de hongos dematiáceos (Fonsecaea pedrosoi, Phialophora verrucosa, Cladophialophora carrionii, entre otros) $)^{1}$. Taxonómicamente este grupo de hongos son clasificados en la clase Ascomycetes, orden Chaetothyriales, familia Herpotrichiellaceae y se caracterizan por presentar estructuras microscópicas multicelulares gruesas coloreadas, llamadas muriformes o cuerpos fumagoides ${ }^{1,2}$.

La respuesta inmune está mediada por neutrófilos y macrófagos causando procesos granulomatosos ${ }^{3}$. Los macrófagos no son capaces de destruir las células fúngicas fagocitadas y éstas se desarrollan en su interior. Según la respuesta inmune dominante se pueden diferenciar cinco formas clínicas: nodulares, lesiones tumorales, verrugosa, placa y cicatriciales ${ }^{4}$; pudiendo ser formas leves, como pequeños nódulos ${ }^{5}$, hasta enfermedades incapacitantes extensas ${ }^{6,7}$.

Actualmente se busca un diagnóstico temprano y oportuno que logre diferenciar las micosis subcutáneas de otras enfermedades fúngicas, así como otras enfermedades dermatológicas (leishmaniasis, esporotricosis, psoriasis, lepra, tuberculosis cutánea) para un manejo oportuno ${ }^{2}$. Si no se trata a tiempo, las complicaciones pueden ser infecciones secundarias, ulceraciones y carcinogénesis de células escamosas ${ }^{1,3}$.

Se presentan dos casos clínicos con el fin de contribuir al conocimiento clínico, diagnóstico y tratamiento de la enfermedad.

\section{Casos clínicos}

\section{Caso clínico 1}

Varón con 56 años de edad, agricultor, procedente de Tarapoto, Región San Martín, Perú. Consultó por dolor en la cara ventral de muñeca desde hacía ocho años. Refería prurito y dolor punzante. Se había automedicado con cremas azufradas sin mejoría visible, presentando una cicatrización en la parte más antigua de la lesión (Figura 1a).

En el examen directo con hidróxido de potasio $(\mathrm{KOH})$ al $10 \%$ se observó la presencia de células muriformes o fumagoides de color café con tabique central típica de cromoblastomicosis (Figura 1b). En el cultivo se desarrollaron colonias de $7 \mathrm{~mm}$ a los 10 días, de crecimiento lento, vellosas, limitadas y de color negro, compatibles con un hongo dematiáceo (Figura 1c). El estudio posterior, mediante la técnica de microcultivo y coloración de Ziehl-Neelsen interrumpido ${ }^{8}$ para la caracterización de hifas y conidios, permitió identificar al hongo $F$. pedrosoi (Figura 1d).

Los exámenes de laboratorio complementarios realizados al paciente mostraron una serología negativa para $\mathrm{VIH}$, hematocrito $45 \%$, hemoglobina $14,8 \mathrm{~g} / \mathrm{dL}$, recuento de leucocitos de 7.800 céls $/ \mathrm{mm}^{3}$ y eosinofilia $(12 \%, 936$ céls $/ \mathrm{mm}^{3}$ ).

Inició tratamiento antifúngico con itraconazol 
$100 \mathrm{mg}$ cada $12 \mathrm{~h}$ durante un mes; sin embargo, el paciente no regresó a su control y abandonó el tratamiento médico, perdiendo la posibilidad de observar la evolución clínica.

\section{Caso clínico 2}

Mujer de 48 años de edad, diabética y procedente de Bagua, Región Amazonas, Perú. Consultó por una lesión de más de 10 años que se inició en la zona media del dedo pulgar. Presentaba eritema, prurito, descamación, puntos negros y dolor ocasional en la región distal del pulgar (Figura 2a).

$\mathrm{Al}$ realizar el examen directo con hidróxido de potasio al 10\% se observó presencia de células muriformes sugerentes de cromoblastomicosis (Figura 2b). En el cultivo se observaron colonias radiadas de color verde oscuro de 14 $\mathrm{mm}$ a los 10 días de incubación (Figura 2c) que mediante la técnica de microcultivo y microscopia se identificó Fonsecaea sp. (Figura 2d).

Estudios hematológicos complementarios mostraron un hematocrito $43,2 \%$, hemoglobina $13,8 \mathrm{~g} / \mathrm{dL}$, leucocitos de 7620 céls $/ \mathrm{mm}^{3}$ y $4 \%$ de eosinófilos $\left(305\right.$ céls $\left./ \mathrm{mm}^{3}\right)$. Adicionalmente, las serologías para Chagas, VIH y HTLV resultaron negativas.

La paciente recibió tratamiento con itraconazol $100 \mathrm{mg}$ cada $12 \mathrm{~h}$, durante dos meses. Sin embargo, un cultivo de control demostró viabilidad del hongo en la lesión. La paciente abandonó el tratamiento posterior y controles siguientes, razón por el cual no permitió observar la evolución de la enfermedad.

\section{Discusión}

Se reportan dos casos de cromoblastomicosis con lesiones dérmicas similares, donde la postergación de la atención médica especializada propició un tiempo de enfermedad prolongada (mayor a ocho años). Esta enfermedad de curso crónico es un problema social para la población afectada que, debido a su contexto cultural, busca un tratamiento no médico. Esto puede exacerbar la lesión inicial dificultando el diagnóstico correcto.

Es importante mencionar que estas infecciones ocurren en zonas tropicales y subtropicales de todo el mundo. En Perú sólo se han reportado entre 10 a 49 casos, número similar a lo descrito en Argentina y E.U.A; pero menos que en Venezuela y México. Sin embargo, es en Brasil donde se ha comunicado el mayor número de casos (500 publicados $)^{9}$, con una prevalencia estimada de uno de cada 196.000 habitantes $^{10}$.

La presentación clínica de ambos casos fue en las extremidades superiores, en contraste con un estudio realizado en Brasil, donde la presentación más frecuente fue en los miembros inferiores $(81,5 \%)^{7}$.

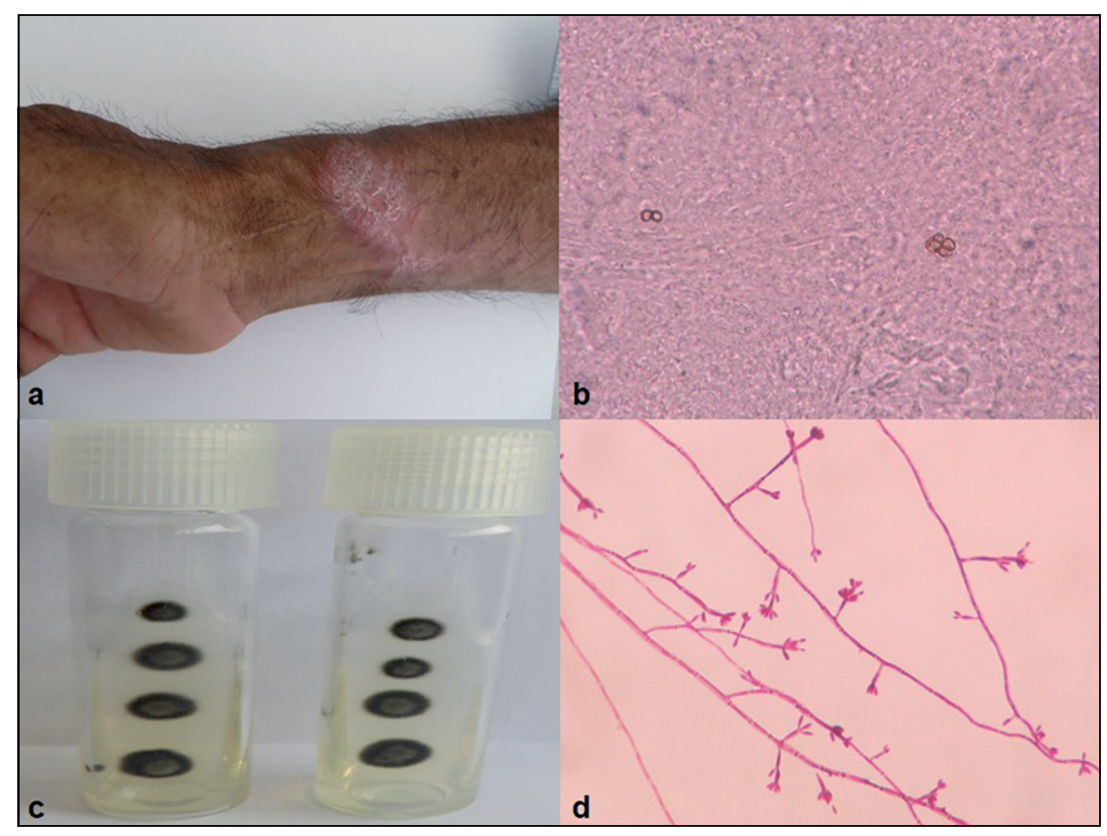

Figura 1. Cromoblastomicosis por Fonsecaea pedrosoi: a) Lesión eritemato-escamosa; b) células muriformes de color café y tabique central (400 aumentos); c) Colonia negruzca a 10 días de incubación en agar Sabouraud; d) Hifas tabicadas con conidios elípticos 400 aumentos.

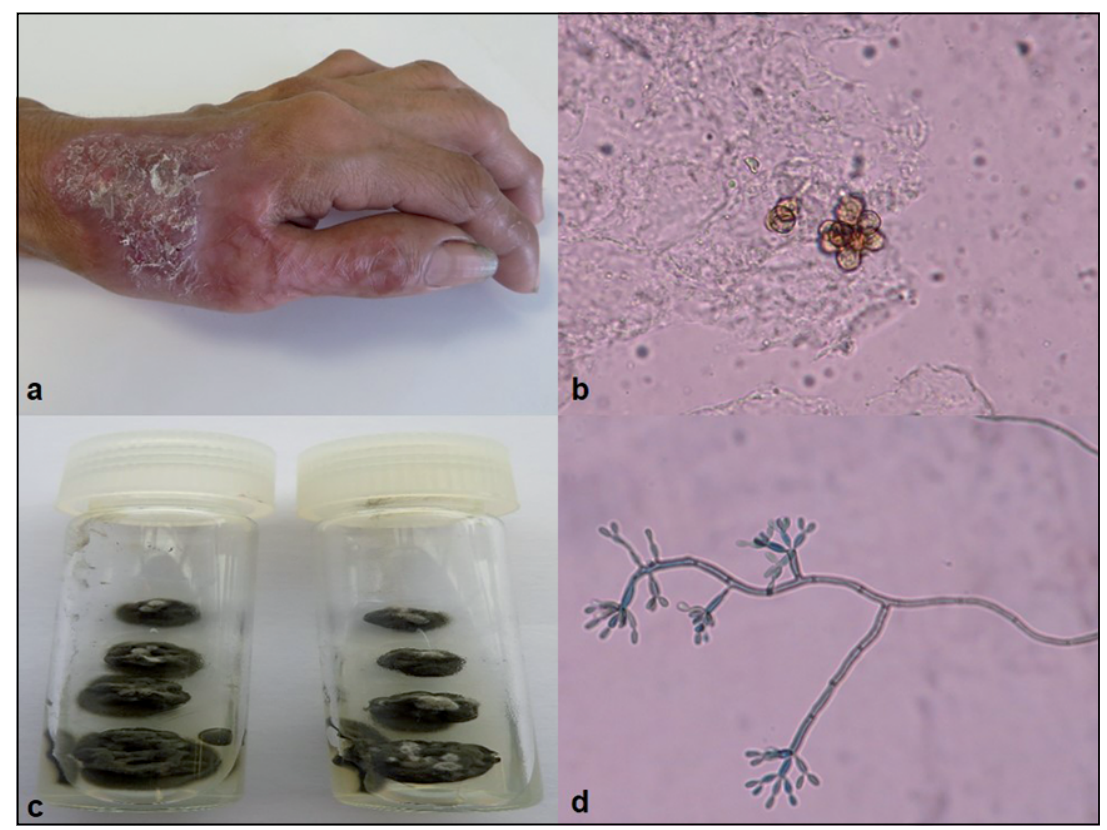

Figura 2. Cromoblastomicosis por Fonsecaea sp: a) Lesión eritemato-escamosa; b) células muriformes a 400 aumentos; c) Colonias radiadas verde oscuras a 10 días de incubación en agar Sabouraud; d) Hifas tabicadas y conidios elípticos a 400 aumentos.

El diagnóstico inicial de los pacientes fueron leishmaniasis cutánea, lo que retrasó el tratamiento oportuno. Sin embargo, a pesar de la cronicidad, postergación de tratamiento específico y que uno de ellos presentaba 
diabetes mellitus como co-morbilidad, ninguno presentó complicaciones. Esto contrasta con casos en Brasil y Perú, donde los pacientes inicialmente diagnosticados como leishmaniasis, presentaron cromoblastomicosis diseminada ${ }^{6,11}$.

La observación de células muriformes ( 5 a 12 um) en muestras clínicas es confirmatorio de cromoblastomicosis. Puede ser diagnosticado por examen microscópico directo con $\mathrm{KOH}$ al $20 \%$ y estudio histopatológico por biopsia. Este último examen tiene la ventaja de mejorar los resultados positivos del cultivo debido a la disminución de la contaminación bacteriana ${ }^{4}$.

En dos años de nuestra práctica clínica encontramos dos casos de cromoblastomicosis, a diferencia de un estudio previo, también en Perú, donde se describieron tres casos en 40 años $^{12}$. La cromoblastomicosis en Perú podría estar subdiagnosticada y su frecuencia subestimada debido a que, no es una enfermedad de reporte obligatorio o de vigilancia epidemiológica. Además, en las regiones donde se presenta la enfermedad, por cuestiones culturales, la población afectada no considera como primera opción la atención médica; y cuando tiene la oportunidad de recibirla, no es diagnosticada con facilidad.

Recomendamos que el diagnóstico microbiológico preliminar se inicie con una adecuada toma de muestra. Para ello se debe prestar mucha atención en la recolección, debiendo realizarse de lesiones cutáneas cubiertas de puntos negros. La muestra deberá evaluarse mediante examen microscópico directo con hidróxido de potasio al $20 \%{ }^{4}$, una técnica sencilla, económica y ampliamente utilizada como tamizaje ${ }^{10}$. El cultivo micológico como estándar de oro, es confirmatorio y permite estudiar a la especie fúngica tanto en la macroscopia como en microscopia.

Los medicamentos con mayor eficacia para el tratamiento de la cromoblastomicosis son itraconazol oral $200 \mathrm{mg} /$ día, durante 6 a 12 meses y terbinafina 250$500 \mathrm{mg} /$ día, durante 12 meses $^{13}$. También se ha descrito terapias combinadas de sesiones mensuales de criocirugía con itraconazol oral ( 300 a $400 \mathrm{mg}$ /día) durante 6 a 8 me$\operatorname{ses}^{14}$. Voriconazol ha sido empleado en dosis similares ${ }^{15}$. Sin embargo, la capacidad intrínseca de $F$. pedrosoi de producir melanina como factor de virulencia contribuye a la reducida susceptibilidad a muchos antimicóticos ${ }^{16}$.

En conclusión, se describen dos casos clínicos de cromoblastomicosis procedentes de zonas tropicales del norte del Perú, causado por F. pedrosoi y Fonsecaea sp. El correcto y oportuno diagnóstico clínico y de laboratorio son necesarios para iniciar un tratamiento adecuado que permita recuperar la calidad de vida de las personas afectadas con esta enfermedad desatendida en el Perú.

Agradecimientos. Al servicio de Infectología del Hospital Regional Lambayeque, Chiclayo, Perú. Asimismo al médico infectólogo Miguel Villegas Chiroque por su valiosa colaboración para el acceso a los pacientes e historias clínicas.

\section{Resumen}

La cromoblastomicosis es una enfermedad fúngica desatendida de curso crónico, que infecta por inoculación al tejido subcutáneo de personas con riesgo ocupacional. Comunicamos dos casos de adultos procedentes de regiones tropicales del norte del Perú, afectados con lesiones crónicas eritemato-escamosas y puntos negros múltiples, con dolor, prurito y cicatrización en áreas más antiguas de la lesión. El diagnóstico se realizó con el examen directo de escamas dérmicas con presencias de células fumagoides típicas y el aislamiento de hongos dematiáceos Fonsecaea pedrosoi y Fonsecaea sp., en el primer y segundo caso; respectivamente. Los pacientes recibieron tratamiento antifúngico con itraconazol, sin poder observar el resultado final debido al abandono del tratamiento y control médico.

\section{Referencias bibliográficas}

1.- Krzyściak P M, Pindycka-Piaszczyńska M, Piaszczyński M. Chromoblastomycosis. Postępy Dermatol Alergol 2014; 31: 310-21.

2.- Raj H J, Majumdar B, Jain A, Maiti P K, Chatterjee G. A clinic- mycological study on suspected cases of chromoblastomycosis: challenges in diagnosis and management. J Clin Diagn Res 2015; 9: WC01-4.

3.- Santos A L, Palmeira V F, Rozental S, Kneipp L F, Nimrichter L, Alviano D S, et al. Biology and pathogenesis of Fonsecaea pedrosoi, the major etiologic agent of chromoblastomycosis. FEMS Microbiol Rev 2007; 31: 570-91.

4.- Queiros-Tellez F, Esterre P, Pérez-Blanco
M, Vitale R G, Salgado C G, Bonifaz A. Chromoblastomycosis: an overview of clinical manifestations, diagnosis and treatment. Med Mycol 2009; 47: 3-15.

5.- Kim D M, Hwang S M, Suh M K, Ha G Y, Choi G S, Shin J, et al. Chromoblastomycosis caused by Fonsecaea pedrosoi. Ann Dermatol 2011; 23: 369-74.

6.- Solórzano S, García R, Hernández-Córdova G. Cromomicosis: reporte de un caso incapacitante. Rev Peru Med Exp Salud Pública 2011; 28; 552-5.

7.- Pires C A, Xavier M B, Quaresma J A, Macedo G M, Sousa B R, Brito A C. Clinical, epidemiological and mycological report on 65 patients from the Eastern Amazon region with chromoblastomycosis. An Bras Dermatol 2012; 87: 555-60.

8.- Ventura R. Ziehl Neelsen interrumpido para coloración de estructuras fúngicas. Rev Exp Med 2015; 1(1): 30-1.

9.- Queiroz-Telles F. Chromoblastomycosis: a neglected tropical disease. Rev Inst Med Trop Sao Paulo 2015; 57 Suppl 19: 46-50.

10.- Marques G F, Masuda P Y, Sousa J M, Barreto J A, Wachholz P A. Clinical and demographic profile of chromoblastomycosis in a referral service in the midwest of São Paulo state (Brazil). An Bras Dermatol 2015; 90:140-2.

11.- Gomes N M, Bastos T C, Cruz K S, 
Francesconi F. Chromoblastomycosis: an exuberant case. An Bras Dermatol 2014; 89 : 351-2.

12.- Burstein A Z. Cromomicosis: Clínica y tratamiento; situación epidemiológica en Latinoamérica. Rev Perú Med Exp Salud Pública 2004; 21: 167-75.

13.- Alvarez-Montiel I, Bonifaz A.
Cromoblastomicosis en placa superficial. Manifestación de una variante poco habitual. Dermatol Rev Mex 2014; 58: 529-33.

14.- Torres-Guerrero E, Isa-Isa R, Isa M, Arenas R. Chromoblastomycosis. Clin Dermatol 2012; 30: 403-8.

15.- Criado P R, Careta M F, Valente N Y, Martins J E, Rivitti E A, Spina R, et al. Extensive long-standing chromomycosis due to Fonsecaea pedrosoi: three cases with relevant improvement under voriconazole therapy. J Dermatolog Treat 2011; 22: 167-74.

16.- Urán M E, Cano L E. Melanina: implicaciones en la patogénesis de algunas enfermedades y su capacidad de evadir la respuesta inmune del hospedero. Infectio 2008; 12: 128-48. 